\title{
Node Throughput Analysis of Decentralized Wireless Networks Using Multibeam Antennas in Multipath Environments
}

\author{
Xin Li • Yimin Zhang • Moeness G. Amin
}

Published online: 26 September 2008

C) Springer Science+Business Media, LLC. 2008

\begin{abstract}
The use of multibeam antennas (MBAs) in decentralized wireless networks significantly increases the throughput by improving spatial reuse and extending coverage compared with single-beam antennas. The throughput performance may, however, degrade in multipath environments due to the fact that multipath propagation increases the probability of collision among different users. Based on the probability of collision, accurate analytical expressions are derived for the node throughput gain (NTG) of a wireless network using MBAs in quasi-stationary multipath environments. The results show that a higher number of propagation paths cause more frequent collisions and thus yield lower node throughput.
\end{abstract}

Keywords Decentralized wireless networks · Directional antenna $\cdot$ Multipath · Throughput gain

\section{Introduction}

Conventional decentralized wireless networks, such as mobile ad hoc and mesh networks, use omnidirectional antennas, which reserve the spectrum over a large area and waste network resources $[1,2]$. It has been shown that directional antennas may mitigate this problem and provide spatial reuse [2]. To further enhance the network capacity and overcome problems like deafness, the use of directional antennas has been extended to multibeam antennas (MBAs) [3-6]. These structures achieve concurrent communications with multiple

X. Li · Y. Zhang $(\varangle) \cdot$ M. G. Amin

Center for Advanced Communications,

Villanova University, Villanova, PA 19085, USA

e-mail: yimin.zhang@villanova.edu

X. Li

e-mail: xin.1@villanova.edu

M. G. Amin

e-mail: moeness.amin@villanova.edu 
neighboring users by forming multiple pre-defined beams or using multiple radios where each radio is equipped with its own directional antenna. Such structures can be optimized in a stationary propagation environment to provide higher network throughput.

The throughput performance of the MBAs can, however, degrade in a multipath propagation environment due to increased probability of collision among different user signals (e.g., $[3,4])$. Multipath propagation is a typical problem in most wireless systems. Specifically, the multipath propagation phenomenon may be more significant in ad hoc networks, since the nodes are typically located in indoor or low-altitude outdoor environments [1]. As such, signals transmitted from a neighboring node may fall into multiple beams at the target node (TN) and result in more frequent collisions.

Previous research on the capacity/throughput of wireless networks mainly focused on the omnidirectional antenna-based networks $[7,8]$ and smart antenna-based networks $[9,10]$. To our best knowledge, there are no analytical throughput expressions for decentralized networks, such as ad hoc networks, exploiting MBAs operating in a multipath environment. In this paper, we consider one-hop throughput of the TN in terms of the node throughput gain (NTG), which is defined as the mean number of successful active neighboring nodes with which a TN can concurrently communicate, from the perspective of the probability of collision. Accurate expressions of the NTG in a quasi-stationary multipath environment are derived and the effect of multipath propagation is analytically evaluated. The obtained results can be conveniently used for an arbitrary number of beams and any number of paths. Simulation examples are provided to demonstrate the accuracy of the analytical expressions and depict the significance of the effect of multipath propagation on the NTG performance.

\section{System Model}

Without loss of generality, it is assumed that a TN has $N$ neighboring nodes that are independently and randomly located around it with a uniform angular distribution. The slotted ALOHA protocol is considered at the medium access control (MAC) layer. Due to random transmission of each node, collision is inevitable [11]. Assume that the probability with which a neighboring node starts packet transmission is $p$, where the packets could be either newly arrived or retransmitted ones. The former follows a Poisson distribution with arrival rate $\lambda$ packets/s. Consequently, during a time slot, there are $n \leq N$ neighboring nodes attempting to send packets to the TN. For the convenience of analysis, we use a simple sector-based model which is similar to that used in [6] (A more realistic antenna model can also be considered). In this model, a considered TN is equipped with $M$ fixed-beam antennas, each forming a pre-defined conical beam spanning a sector of $2 \pi / M$ radians. As such, the $M$ antennas form $M$ non-overlapping beams which collectively cover the entire azimuth plane. The beam shape is assumed to be conical, i.e., the antenna gain within the beam sector is constant whereas it drops to zero outside the beam sector.

Depending on the applications and environments, wireless communication channels often experience multipath propagation. The number of paths and their distributions vary as the propagation environment changes. In this paper, it is assumed that a channel is composed of several dominant paths, each with resolvable angle-of-arrival (AOA) and sufficiently high strength such that each path warrants signal reception if it does not compete with other signal arrivals in the same beam, or is collided with other signals if they fall into the same beam. The multipath components are assumed to be quasi-stationary so that they are time-invariant over a packet. 


\section{Node Throughput Gain Analysis}

\subsection{Single-Path Propagation Environment}

In a single-path propagation environment, it is clear that a collision occurs within a beam when more than one active neighboring users fall into the beam [3,12]. As such, the probability of having collisions in a beam can be evaluated. The probability of concurrent packet reception for a beam has been considered in [6]. In this paper, we consider another capacity metric, namely the node throughput gain (NTG), denoted as $G$ [3-5]. As we addressed earlier, the NTG is defined as the mean number of successful active neighboring nodes with which a TN can concurrently communicate. This metric allows us to focus on the effect of some relevant MAC layer parameters rather than physical layer parameters. As such, it reflects not only the advantage of the MBAs over omnidirectional antennas and single-beam directional antennas, but also the efficiency of the employed MAC protocols. For a specific application, the NTG can be mapped to the actual data rate based on the information of modulation and coding schemes.

We first define $P_{n}^{\mathrm{a}}(n, N, p)$ as the probability that, during a time slot, $n$ neighboring nodes occur around a TN and attempt to make transmissions. This probability can be described as the following binomial distribution

$$
P_{n}^{\mathrm{a}}(n, N, p)=\left(\begin{array}{l}
N \\
n
\end{array}\right) p^{n}(1-p)^{N-n},
$$

where $\left(\begin{array}{l}N \\ n\end{array}\right)$ denotes the combination operation, representing the number of different ways of selecting $n$ out of $N$ neighboring users. Further, we define $P_{n}^{\mathrm{s}}(n, 1, M)$ as the probability that one user is successfully received by the TN in the presence of $n$ active neighboring nodes in a single path propagation environment, i.e., one beam is occupied by the signal transmitted from a single neighboring user. In this case, the remaining $M-1$ beams can be occupied arbitrarily by other $n-1$ active users, and hence one can find

$$
P_{n}^{\mathrm{s}}(n, 1, M)=\left(1-\frac{1}{M}\right)^{n-1} .
$$

Then, the NTG in the presence of single path can be written as

$$
G^{\mathrm{sp}}=\sum_{n=1}^{N} n P_{n}^{\mathrm{a}}(n, N, p) P_{n}^{\mathrm{s}}(n, 1, M) .
$$

The superscript 'SP' is used to emphasize the single-path propagation environment.

For a constant $N p$, the binomial model depicted in (1) becomes a Poisson distribution as $p \rightarrow 0$. The equivalent traffic load in the Poisson distribution, which is the product of the packet arrival rate and packet duration, can be computed as $R=N p$. Thus, (1) can be approximated as

$$
P_{n}^{\mathrm{a}}(n, R)=\frac{R^{n} e^{-R}}{n !} .
$$

Substituting (4) into (3) yields

$$
G^{\mathrm{sp}}=R e^{-R / M},
$$


which is consistent with the result given in [5]. It is evident that the maximum NTG is $M / e$, which is achieved when $R=N p=M$. That is, in a single-path propagation environment, the maximum NTG is obtained when the mean number of active neighboring users equals the number of available beams.

\subsection{Multipath Propagation Environment}

As we pointed out earlier, a decentralized network is typically located in an environment with rich multipath propagation. In this situation, a signal transmitted from a neighboring node may arrive at the TN through multiple propagation paths and fall into multiple beams. As such, more frequent collisions are likely to occur. Because all nodes are located in a similar multipath environment, their propagation channels to the TN are similar. For the convenience of analysis, therefore, it can be assumed that, during a time slot, the respective signal transmitted from each active neighboring node arrive at the TN through $K \geq 1$ quasi-stationary propagation paths, each with resolvable AOA and sufficient high strength, as discussed in Sect. 2. As such, the signal arrivals from each active neighboring user may fall into at most $B=\min (K, M)$ beams.

In a multipath propagation environment, a neighboring node $\mathrm{u}_{i}$ is considered collided if all the beams occupied by the paths of $\mathrm{u}_{i}$ collide, i.e., none of $\mathrm{u}_{i}$ 's paths is free of collision. As such, the NTG in a multipath propagation environment can be expressed as

$$
G^{\mathrm{mp}}=\sum_{n=1}^{N} n P_{n}^{\mathrm{a}}(n, N, p) P_{n}^{\mathrm{s}}(n, K, M),
$$

where $P_{n}^{\mathrm{a}}(\cdot)$ is given in (1) and can be approximated as (4), and $P_{n}^{\mathrm{s}}(n, K, M)$ has a similar definition as (2) except that each transmission now has $K$ quasi-stationary paths. The latter can be obtained from the following equation,

$$
P_{n}^{\mathrm{s}}(n, K, M)=1-P_{n}^{\mathrm{c}}(n, K, M),
$$

where $P_{n}^{\mathrm{c}}(n, K, M)$ is the probability of collision, which is defined as the probability that multiple active users fall into the same beam and hence collide. According to this definition, we can obtain

$$
P_{n}^{\mathrm{c}}(n, K, M)=\sum_{b=1}^{B} P_{b}^{\mathrm{b}}(b, K, M) P_{b}^{\mathrm{c}}(b, n, K, M),
$$

where $P_{b}^{\mathrm{b}}(\cdot)$ is the probability that all paths originating from one user fall into $b$ beams of the $\mathrm{TN}$, and $P_{b}^{\mathrm{c}}(\cdot)$ is the collision probability that collisions occur within all the $b$ beams. From the probability theory and after some mathematic operations, we can obtain

$$
P_{b}^{\mathrm{b}}(b, K, M)={ }^{M} P_{b} \frac{\mathrm{S}(K, b)}{M^{K}},
$$

and

$$
P_{b}^{\mathrm{c}}(b, n, K, M)=1-\sum_{i=1}^{b}(-1)^{i+1}\left(\begin{array}{l}
b \\
i
\end{array}\right)\left(1-\frac{i}{M}\right)^{K(n-1)},
$$

respectively, where ${ }^{M} P_{b}$ denotes the permutation operation, representing the number of ways of selecting $b$ beams from all $M$ available beams, and $\mathrm{S}(K, b)=\frac{1}{b !} \sum_{i=0}^{b-1}(-1)^{i}\left(\begin{array}{l}b \\ i\end{array}\right)(b-i)^{K}$ 
is the Stirling number of the second kind [13], representing the number of different ways that $K$ paths occupy $b$ beams. The derivation of (10) is given in Appendix.

Substituting (9) and (10) into (8) and applying the fact $\sum_{b=1}^{B} P_{b}^{\mathrm{b}}(b, K, M)=1$, one can further obtain

$$
P_{n}^{\mathrm{c}}(n, K, M)=1-\sum_{b=1}^{B} P_{b}^{\mathrm{b}}(b, K, M) \sum_{i=1}^{b}(-1)^{i+1}\left(\begin{array}{l}
b \\
i
\end{array}\right)\left(1-\frac{i}{M}\right)^{K(n-1)} .
$$

Thus, (7) can be rewritten as

$$
P_{n}^{\mathrm{S}}(n, K, M)=\sum_{b=1}^{B} P_{b}^{\mathrm{b}}(b, K, M) \sum_{i=1}^{b}(-1)^{i+1}\left(\begin{array}{l}
b \\
i
\end{array}\right)\left(1-\frac{i}{M}\right)^{K(n-1)} .
$$

It is easy to find that, for $K=1,(12)$ is reduced to (2). Further, substituting (12) into (6) yields

$$
G^{\mathrm{mp}}=\sum_{n=1}^{N} n P_{n}^{\mathrm{a}}(n, N, p) \sum_{b=1}^{B} P_{b}^{\mathrm{b}}(b, K, M) \sum_{i=1}^{b}(-1)^{i+1}\left(\begin{array}{l}
b \\
i
\end{array}\right)\left(1-\frac{i}{M}\right)^{K(n-1)} .
$$

Substituting (1) and (9) into (13), the complete formula for $G^{\mathrm{mp}}$ is obtained as

$$
\begin{aligned}
G^{\mathrm{mp}}= & \frac{1}{M^{K}} \sum_{n=1}^{N} n\left(\begin{array}{l}
N \\
n
\end{array}\right) p^{n}(1-p)^{N-n} \sum_{b=1}^{B}\left(\begin{array}{l}
M \\
b
\end{array}\right) \\
& \times \sum_{j=0}^{b-1}(-1)^{j}\left(\begin{array}{l}
b \\
j
\end{array}\right)(b-j)^{K} \sum_{i=1}^{b}(-1)^{i+1}\left(\begin{array}{l}
b \\
i
\end{array}\right)\left(1-\frac{i}{M}\right)^{K(n-1)} .
\end{aligned}
$$

The results of the above equation can be easily evaluated using numerical computations since $B$ and $M$ are relatively small. Numerical results shown that (14) is a decreasing function of $K$ and an increasing function of $M$. That is, multipath propagation is always detrimental to the enhancement of node throughput, whereas the node throughput benefits from the increasing number of available beams at the TN.

\section{Numerical and Simulation Results}

Numerical and simulation results are provided to verify the accuracy of the analytical expressions and to depict the significance of the effect of multipath propagation. It is assumed that, during a time slot, each neighboring node attempts to start a packet transmission with probability $p=0.1$. The multipath model given in Sect. 2 is considered, and the number of propagation paths $(K)$ varies from 1 to 6 . The offered load $(R)$ varies from 0 to 20 (i.e., the corresponding number of neighboring nodes varies from 0 to 200). Each simulation result is obtained using 1,000 consecutive time slots. In each time slot, the active neighboring nodes and their respective propagation paths are randomly and independently generated.

The analytical and simulation results of the NTG in a quasi-stationary multipath environment are plotted in Fig. 1a, b, respectively, for four $(M=4)$ and eight $(M=8)$ beam scenarios. Both figures clearly show good consistence between the simulation and analytical results. The results also confirm the discussion we made in Sect. 3.2 that the performance of MBAs degrades in a multipath propagation environment. Such performance degradation is 
Fig. 1 Analytical (lines) and simulated (markers) node throughput gain in various multipath environments. a Target node with four beams. b Target node with eight beams
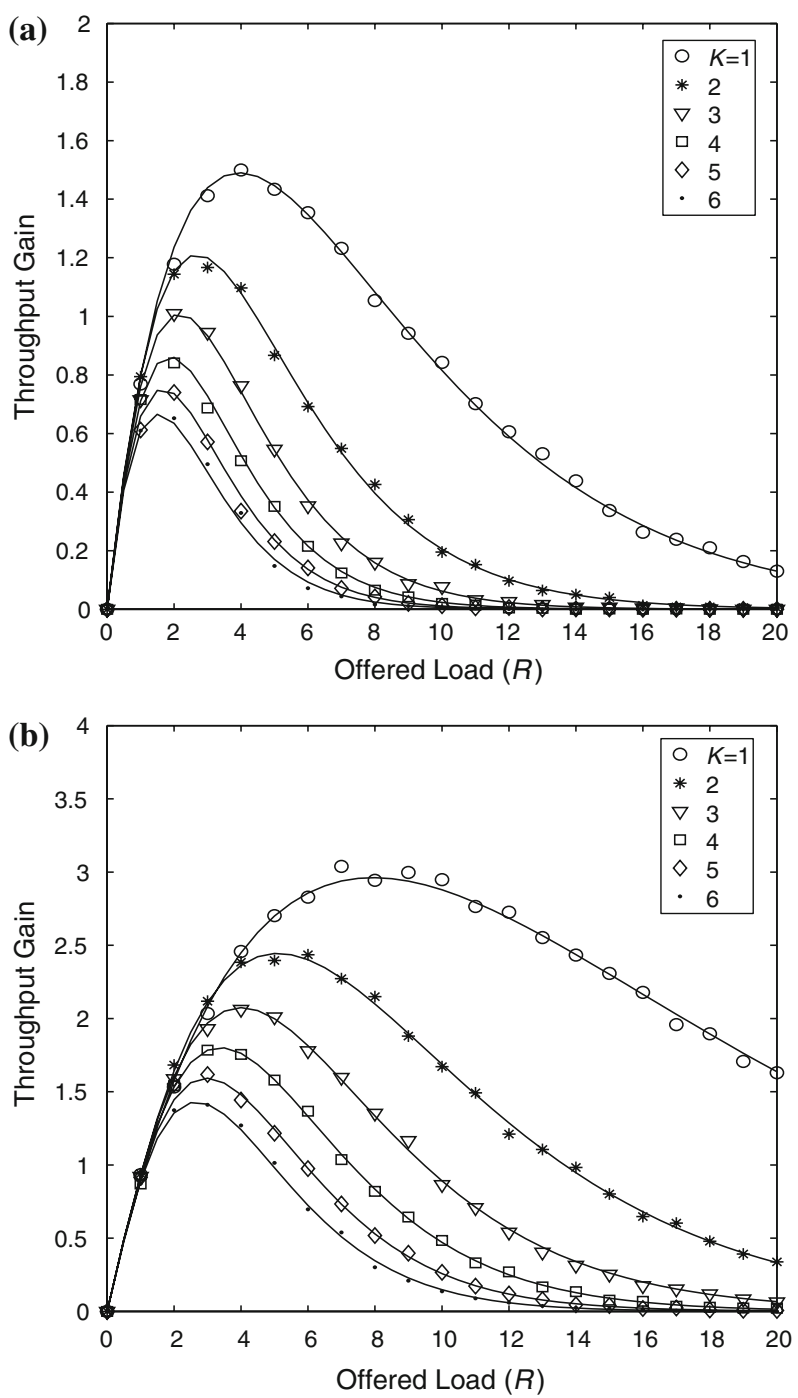

more significant when the offered load is high, since in such a scenario the probability of collision among different user signals is increased.

As a result of random transmission from the neighboring users, the NTG is not a monotonic function of the offered load. Once the maximum NTG $G_{\max }$ is reached, further increasing the offered load results in reduced node throughput. As the number of paths $K$ increases, $G_{\max }$ decreases and corresponds to a lower value of the offered load, because increasing the number of paths or offered load causes more frequent collisions. By comparing the two figures depicted in Fig. 1, it is evident that the NTG increases as the number of beams available at the $\mathrm{TN}$ increases, since a higher number of available beams can concurrently support more users.

The impact of the number of propagation paths on the maximum node throughput gain is illustrated in Fig. 2. As is seen here, $G_{\max }$ is a monotonically decreasing function of the 
Fig. 2 Impact of the number of propagation paths on the maximum node throughput gain

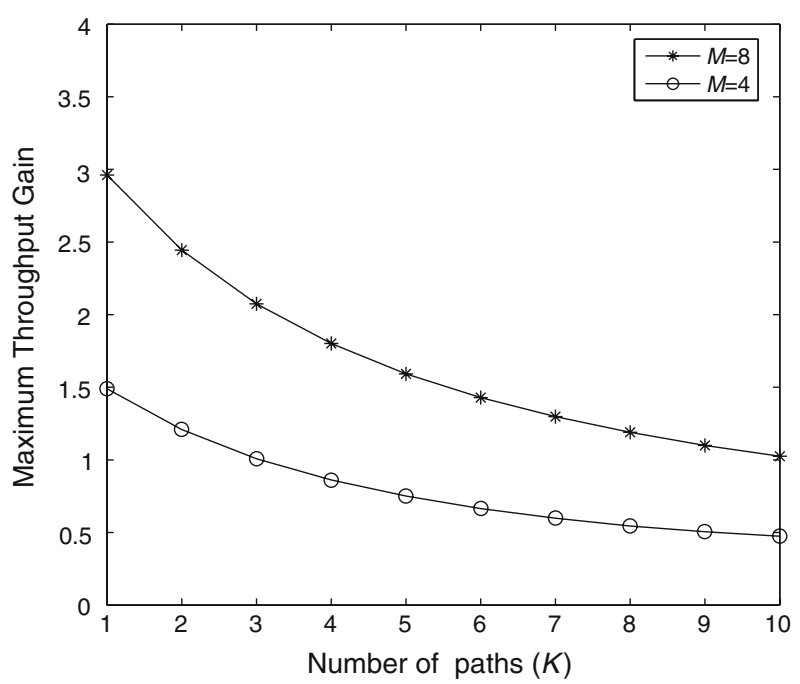

number of paths $K$. That is, more propagation paths yield a lower $G_{\max }$. Furthermore, the slope of the curve decreases as $K$ increase. Consider the fact that $K$ is bounded in practice, a saturated $G_{\max }$ value can be assumed when the number of available beams, $M$, is specified. It is also observed in Fig. 2 that, for a given number of paths, the value of $G_{\max }$ corresponding to $M=8$ is approximately doubled from that corresponding to $M=4$. Thus, increasing the number of available beams is effective to enhance the node throughput and to overcome multipath propagation. For example, the value of $G_{\max }$ in the case of $M=8$ and $K=6$ approaches that in the case of $M=4$ and $K=1$. In this case, the detrimental impact of six-path propagation on the throughput can be compensated at the cost of using additional four beams.

\section{Conclusions}

From the perspective of the probability of collision, this paper has derived general and accurate analytical expressions for the node throughput gain of a decentralized wireless network exploiting multibeam antennas. Both single-path and quasi-stationary multipath environments are considered and the impact of multiple propagation paths is examined. The performance analysis clearly shows that a higher number of propagation paths result in more frequent collisions and consequently a lower node throughput, whereas increasing the number of available beams at the target node provides a higher node throughput. The analytical expressions can be conveniently used to assess the node throughput for an arbitrary number of beams as well as propagation paths.

\section{Appendix}

Let $B_{1}, B_{2}, \ldots, B_{b}$ be $b$ events defined in a probability space, where $B_{j}, j=1, \ldots, b$, denotes the event that the $j$ th beam collides. $\operatorname{Pr}\left\{B_{j}\right\}$ denotes the probability at which event $B_{j}$ occurs. According to the set properties and the inclusion and exclusion principle, one can obtain 


$$
\begin{aligned}
P_{b}^{\mathrm{c}}(b, n, K, M) & =\operatorname{Pr}\left\{B_{1}, B_{2}, \ldots, B_{b}\right\}=\operatorname{Pr}\left\{\bigcap_{j=1}^{b} B_{j}\right\} \\
& =1-\operatorname{Pr}\left\{\bigcup_{j=1}^{b} \overline{B_{j}}\right\},
\end{aligned}
$$

where

$$
\begin{aligned}
\operatorname{Pr}\left\{\bigcup_{j=1}^{b} \overline{B_{j}}\right\}= & \sum_{k=1}^{b} \operatorname{Pr}\left\{\overline{B_{k}}\right\}-\sum_{1 \leq k_{1}<k_{2} \leq b} \operatorname{Pr}\left\{\overline{B_{k_{1}}} \bigcap \overline{B_{k_{2}}}\right\}+\cdots \\
& +(-1)^{i+1} \sum_{1 \leq k_{1}<\cdots<k_{i} \leq b} \operatorname{Pr}\left\{\bigcap_{v=1}^{i} \overline{B_{k_{v}}}\right\}+\cdots \\
& +(-1)^{b+1} \operatorname{Pr}\left\{\bigcap_{k=1}^{b} \overline{B_{k}}\right\} .
\end{aligned}
$$

The number of terms contained in the $i$ th summation, $\sum_{1 \leq k_{1}<\cdots<k_{i} \leq b} \operatorname{Pr}\left\{\bigcap_{v=1}^{i} \overline{B_{k_{v}}}\right\}$, on the right-hand side of (A2) is $\left(\begin{array}{l}b \\ i\end{array}\right)$. Due to the assumption that the neighboring nodes are randomly located around the $\mathrm{TN}$ with a uniform angular distribution, we obtain

$$
\begin{gathered}
\operatorname{Pr}\left\{\overline{B_{k}}\right\}=\left(1-\frac{1}{M}\right)^{K(n-1)}, k=1, \ldots, b, \\
\operatorname{Pr}\left\{\bigcap_{v=1}^{i} \overline{B_{k_{v}}}\right\}=\left(1-\frac{i}{M}\right)^{K(n-1)}, k_{v} \in\{1, \ldots, b\}, \quad i=1, \ldots, b .
\end{gathered}
$$

Subsequently, (10) can be derived straightforwardly by first substituting (A3) and (A4) into (A2) and then into (A1).

Acknowledgements This work was supported in part by the NSF under grant No. EEC-0332490.

\section{References}

1. Winters, J. H. (2006). Smart antenna techniques and their application to wireless ad hoc networks. IEEE Wireless Communication, 13(4), 77-83.

2. Bandyopadhyay, S., Roy, S., \& Ueda, T. (2006). Enhancing the performance of ad hoc wireless networks with smart antennas. Boca Raton, FL: Auerbach.

3. Zhang, Y., Li, X., \& Amin, M. G. (Oct.-Nov. 2006). Multi-channel smart antennas in wireless networks. In Asilomar Conference on Signals, Systems, and Computers, Pacific Grove, CA.

4. Li, X., Zhang, Y., \& Amin, M. G. (June 2007). Performance of wireless networks exploiting multi-channel smart antennas in multipath environments. In International Waveform Diversity and Design Conference, Pissa, Italy.

5. Chockalingam, A., \& Rao, R. R. (1998). MAC layer performance with steerable multibeam antenna arrays. IEEE PIMRC, 2, 973-977.

6. Jain, V., \& Agrawal, D. P. (2006). Concurrent packet reception bounds for on-demand MAC protocols for multiple beam antennas. IEEE PIMRC. doi:10.1109/PIMRC.2006.254207.

7. Li, J., Blake, C., Decouto, D. S. J., Lee, H. I., \& Morris, R. (2001). Capacity of ad hoc wireless networks. In ACM MobiCom, Rome, Italy. 
8. Toumpis, S., \& Goldsmith, A. J. (2003). Capacity regions for wireless ad hoc networks. IEEE Transactions on Wireless Communication, 2(4), 736-748.

9. Spyropoulos, A., \& Raghavendra, C. S. (2003). Asympotic capacity bounds for ad-hoc networks revisited: The directional and smart antenna cases. IEEE GlobeCom, 3, 1216-1220.

10. Babich, F., Comisso, M., D’Orlando, M., \& Mania, L. (2006). Interference mitigation on WLANs using smart antennas. Wireless Personal Communication Journal, 36(4), 387-401.

11. Ramanathan, R., Redi, J., \& Santivanez, C. (2005). Ad hoc networking with directional antennas: a complete system solution. IEEE Journal of Selected Areas in Communications, 23(3), 496-506.

12. Singh, A., Ramanathan, P., \& Veen, B. V. (2005). Spatial reuse through adaptive interference cancellation in multi-antenna wireless networks. IEEE GlobeCom, 5, 3092-3096.

13. Graham, R. L., Knuth, D. E., \& Patashnik, O. (1994). Concrete mathematics: A foundation for computer science (2nd ed.). MA: Addison-Wesley.

\section{Author Biographies}

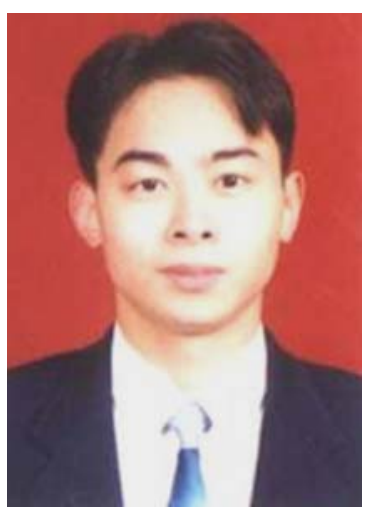

Xin Li was born in Sichuan, China, in 1975. He received the B.S. Degree in Electrical Engineering, and M.S. Degree in Information and Communications Engineering from Chongqing University of Posts and Telecommunications (CQUPT), Chongqing, China, in 1999 and 2002, respectively. He received his $\mathrm{Ph} . \mathrm{D}$. Degree in Information and Communications Engineering \& Electromagnetic Theory from University of Electronic Science and Technology of China (UESTC) in 2005. From December 1999 to May 2001, as a research engineer, he was doing his master's thesis in Datang Technology Co., Ltd., in Beijing, where he participated in drafting and amending the $3 \mathrm{G}$ time division synchronous code division multiple access (TD-SCDMA) standard, and in prototyping the TD-SCDMA/GSM system. From January to July of 2002, he was a research engineer in Holley Co., Ltd., where he developed the dual-mode handset in cooperation with the R\&D department in Dallas, USA. From July 2005 to August 2006, he worked at Alcatel Shanghai Bell Research \& Innovation Center as a research scientist, where he took part in the prototyping of MIMO-OFDM systems for future 4G systems. He is currently a postdoctoral research fellow at the Center for Advanced Communications in Villanova University, USA. His research interests include MIMO radio transmission technologies, OFDM, CDMA, wireless channel modeling, diversity, smart antennas, advanced signal processing and applications, communication network, ad hoc and mesh networks, and circuit and antenna design. He has published over 40 papers.

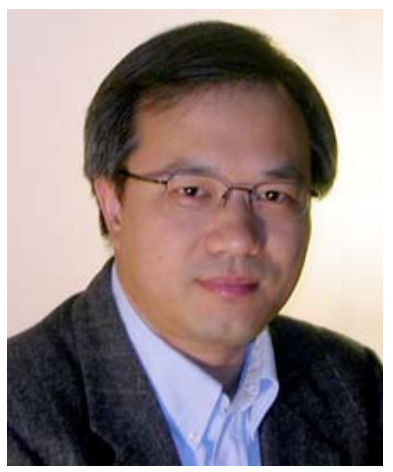

Yimin Zhang received his Ph.D. Degree from the University of Tsukuba, Tsukuba, Japan, in 1988. He joined the faculty of the Department of Radio Engineering, Southeast University, Nanjing, China, in 1988. He served as a Technical Manager at the Communication Laboratory Japan, Kawasaki, Japan, from 1995 to 1997, and was a Visiting Researcher at ATR Adaptive Communications Research Laboratories, Kyoto, Japan, from 1997 to 1998. Since 1998, he has been with the Villanova University, Villanova, PA, where he is currently a Research Associate Professor with the Center for Advanced Communications. Dr. Zhang's research interests lie in the area of statistical signal and array processing for communications and radar systems, including spacetime adaptive processing, wireless networks, MIMO systems, cooperative diversity, blind signal processing, digital mobile communications, time-frequency analysis, source localization and tracking, radar imaging, image processing, and RFID. Dr. Zhang is a Senior Member of IEEE. He serves as an Associate Editor for IEEE Signal Processing Letters and an Associate Editor for the Journal of the Franklin Institute. 


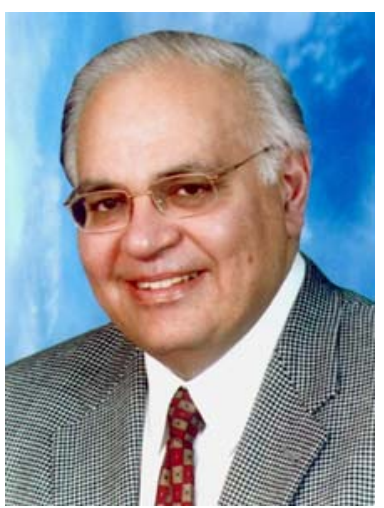

Moeness G. Amin received his Ph.D. Degree in 1984 from University of Colorado, Boulder. He has been on the Faculty of Villanova University since 1985, where is now a Professor in the Department of Electrical and Computer Engineering and the Director of the Center for Advanced Communications. Dr. Amin has over 300 publications in the areas of wireless communications, time-frequency analysis, smart antennas, interference cancellation in broadband communication platforms, direction finding, over the horizon radar, radar imaging, and channel equalizations. Dr. Amin is a fellow of the IEEE and a fellow of the International Society of Optical Engineers; Recipient of the IEEE Third Millennium Medal; Distinguished Lecturer of the IEEE Signal Processing Society for 2003; Member of the Franklin Institute Committee on Science and the Arts; Recipient of the 1997 Villanova University Outstanding Faculty Research Award; Recipient of the 1997 IEEE Philadelphia Section Service Award. Dr. Amin was the Technical Chair of the second IEEE International Symposium on Signal Processing and Information Technology, Morocco, 2002; The General and Organization Chair of the IEEE Workshop on Statistical Signal and Array Processing, Pennsylvania, 2000; The General and Organization Chair of the IEEE International Symposium on Time-Frequency and Time-Scale Analysis, Pennsylvania, 1994. He was an Associate Editor of the IEEE Transactions on Signal Processing during 1996-1998. Dr. Amin was a member of the IEEE Signal Processing Society Technical Committee on Signal Processing for Communications during 1998-2002 and was a Member of the IEEE Signal Processing Society Technical Committee on Statistical Signal and Array Processing during 1995-1997. 